\title{
Fundamental Law of Information: Proved by Both Numbers and Characters in Conjugate Matrices ${ }^{\dagger}$
}

\author{
Xiaohui Zou ${ }^{1,2, *}$, Shunpeng Zou ${ }^{1,2}$ and Lijun Ke ${ }^{2}$ \\ 1 China University of Geosciences, Beijing 100083, China \\ 2 Searle Research Center, University of California Berkeley, Berkeley, CA 94720, USA \\ * Correspondence: zouxiaohui@pku.org.cn; Tel.: +86-159-1624-1709 \\ † Presented at the IS4SI 2017 Summit DIGITALISATION FOR A SUSTAINABLE SOCIETY, Gothenburg, \\ Sweden, 12-16 June 2017.
}

Published: 8 June 2017

\begin{abstract}
Its purpose is to prove information law by logic, mathematics and translation. The method involves: the generalized bilingual logic established on both Aristotle's formal logic and Frege's mathematical logic, the linkage function established on both Turing' strong artificial intelligence using numbers and Searle's weak artificial intelligence using characters, the ontological knowledge established on both Saussure's general linguistics and Chomsky's formal linguistics. The result is that the basic law can be proved by digital and textual twin matrices. Its significance lies in that the global positioning system should be regarded as a special case of the generalized bilingual system.
\end{abstract}

Keywords: information law; bilingual logic; linkage function; ontological knowledge

\section{Introduction}

The purpose of this paper is to prove the fundamental law of information in three verifiable ways: by logic, mathematics and translation. That involves: the basis of both Aristotle's formal logic based on language [1] and Frege's mathematical logic based on arithmetic [2], the basis of both Turing's strong artificial intelligence view based on digital computation [3] and Searle's weak artificial intelligence view based on natural language [4], the basis of both common knowledge [5] and expert knowledge [6] ontologically with interdisciplinary, cross-field and cross-industry, on the basis of both Saussure's general linguistic view [7] and Chomsky's formal linguistic view [8], the reference between English and Chinese and its alternative bilingual [9] to the relation of translation [10].

\section{Materials and Methods}

The main materials or background relates to: the generalized bilingual logic of sequence and position (the premise), the linkage function between digital and textual of conjugate matrices, common knowledge and expert knowledge ontologically with interdisciplinary, cross-field and cross-industry, and English and Chinese and its alternative bilingual. It is to establish the relation of generalized translation based on the ontology of knowledge.

The main specific method of expert knowledge acquisition involves the following steps: The first step, any user can put a piece of text (a standardized expression of expert knowledge) into our sequencing positioning system software, the second step, the software will automatically generate a pair of chessboard generalized bilingual sequencing positioning system, and finally, through Manmachine collaboration, selection and construction of formatted expression of expert knowledge ontology or core terminology framework.

\section{Results}

The result is that the fundamental law can be proved by digital and textual twin matrices. 


\subsection{The Fundamental Law of Information Proved by Both Brain and Computer}

\subsubsection{Proved by Logic}

Representatives:

- Aristotle's formal logic based on language with letters or characters;

- Frege's mathematical logic based on arithmetic with numbers;

- Zou's generalized bilingual logic of sequence and position with both characters and numbers.

The Innovative Knowledge Contributions:

(1) Formal Logic using letters-based deduction for the abstract class;

(2) Mathematical Logic using numbers-based calculation for the case;

(3) Generalized Bilingual Logic using both characters and numbers for the class-case.

There are paradoxes in the practical application of formal logic and mathematical logic. The practical application of the Generalized Bilingual Logic or sequencing positioning system logic does not reserve the opportunity for paradox of human thought. Because, whether it is the overall superchessboard, or the specific twin-chessboards for storing characters and numbers, those lattices on the logical relationship and its structure of the sequence and the location, are unique and constant or conservation.

\subsubsection{Proved by Mathematics}

Representatives:

- Turing's Strong Artificial Intelligence based on Digital Computation;

- Searle's Weak Artificial Intelligence based on Natural Language;

- Zou's Generalized Intelligence based on Bilingual Mathematics.

The Innovative Knowledge Contributions:

(1) Strong Artificial Intelligence based on Digital Computation by using numbers;

(2) Weak Artificial Intelligence based on Natural Language by using letters or characters;

(3) Generalized Intelligence based on Bilingual Mathematics by using the both.

If the limit is in the field of arithmetic computing, then, the Turing computer does exceed the human brain's computing and memory ability; if limited to the field of language communication, then, Searle's Chinese room topic does point out the logic paradox of Turing test. Therefore, Xiaohui believes that: in the field of arithmetic and language common point of view, the linkage function can solve this problem by using the generalized bilingual mathematics. The two broad categories of formal strategies can resolve these two extreme argument problems.

\subsubsection{Proved by Translation}

Representatives:

- Saussure's general linguistics using natural language;

- Chomsky's formal linguistics using formal language;

- Zou's generalized bilingual linguistics using both on surfaces, essentially only arithmetic.

The Innovative Knowledge Contributions:

(1) General Linguistic View for human's natural language understanding;

(2) Formal Linguistic View for computer's natural language understanding;

(3) Generalized Bilingual Linguistic View for both of human brain and computer.

Saussure has clearly pointed out that the object in linguistic is not clear, at the same time, there is a very good argument, chess system and language system is surprisingly connected or the same. Tarski's object language and its interpretation or meta-language division, for the birth of formal 
language, played a very critical role. Chomsky in "syntactic structure" is very clear in the use of a basic formal model S $=\mathrm{NP}+\mathrm{VP}$ that means sentence is equal to noun phrase and verb phrase. Xiaohui explains object language and meta-language as its basic research object system and derived research object systems by using logical sequence structure and linkage function relationship with the twin formal approach, and further, so as to interpersonal communication and human-computer interaction, providing a very satisfactory communication and negotiation platform - through a series of bilingual conversion can be achieved more satisfactory translation effects and machine translation criteria based on their laws and standards.

\subsection{The Digital and Textual Twin Matrices}

Facts speak louder than words. Through a set of facts, that is, the typical embodiment, everyone can feel that the effect of the expert knowledge acquisition method based on the twin checkerboard is accurate, simple and efficient.

Representatives: Aristotle, Frege, Zou. Innovative Knowledge Contributions: Formal Logic for deduction with class, Mathematical Logic for calculation with case, Generalized Bilingual Logic for both class and case with characters and numbers.

The Chinese version of the above paragraph, through Figure 1 and Table 1, you can verify our language chessboard and its application generated knowledge chess-menu and original chess-soul.

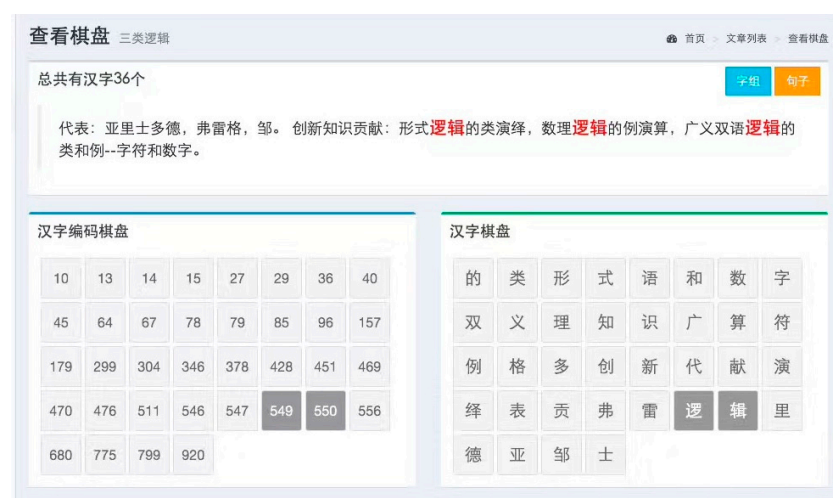

(a)

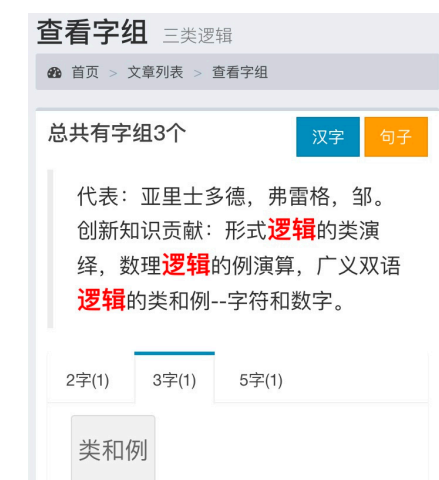

(b)

Figure 1. This is a formal twin chessboard and its application listed as: (a) Description of what is language chessboard; (b) Description of what is knowledge chess-menu and original chess-soul [11].

Table 1. This is a table with chess-menu placed in three rows and columns.

\begin{tabular}{ccc}
\hline Attributes & Concept & Types \\
\hline Formal & Logic & class \\
Mathematical & Logic & Case \\
Bilingual & Logic & class and case \\
\hline
\end{tabular}

Representatives: Turing, Searle, Zou. Innovative Knowledge Contributions: Strong Artificial Intelligence based on Digital Computation by using numbers, Weak Artificial Intelligence based on Natural Language by using alphabet, Generalized Intelligence based on Bilingual Mathematics by using both characters and numbers.

The Chinese version of the above paragraph, through Figure 2 and Table 2, you can verify our language chessboard and its application generated knowledge chess-menu and original chess-soul. 


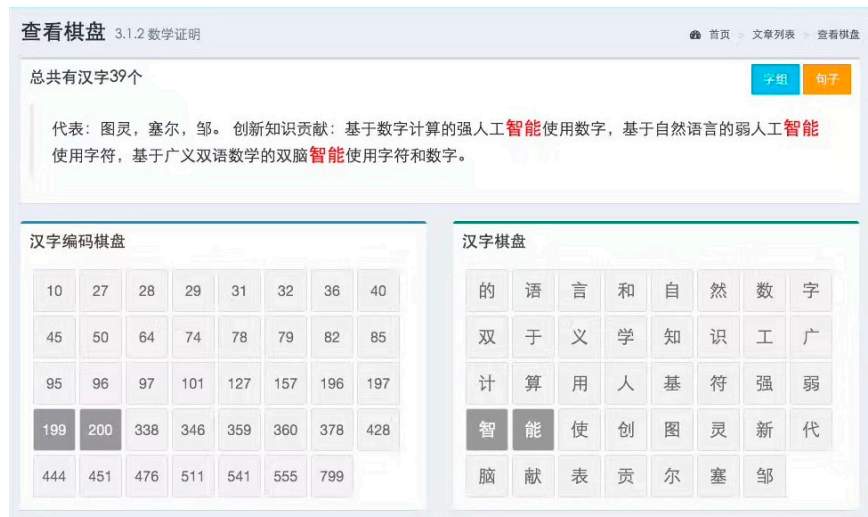

(a)

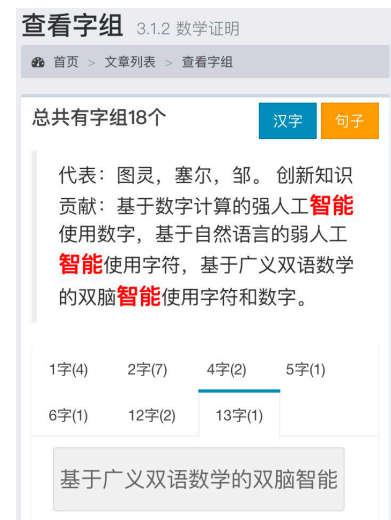

(b)

Figure 2. This is a formal twin chessboard and its application listed as: (a) Description of what is language chessboard; (b) Description of what is knowledge chess-menu and original chess-soul [11].

Table 2. This is a table with chess-menu placed in three rows and columns.

\begin{tabular}{ccc}
\hline Attributes & Concept & Types \\
\hline Artificial & Intelligence & Strong \\
Artificial & Intelligence & Weak \\
Generalized & Intelligence & Bilingual \\
\hline
\end{tabular}

Representatives: Saussure, Chomsky, Zou. Innovative Knowledge Contributions: General Linguistics with human's natural language understanding, Formal Linguistics with computer's natural language understanding, Generalized Bilingual Linguistics with both on surfaces, but essentially only arithmetic.

The Chinese version of the above paragraph, through Figure 3 and Table 3, you can verify our language chessboard and its application generated knowledge chess-menu and original chess-soul.

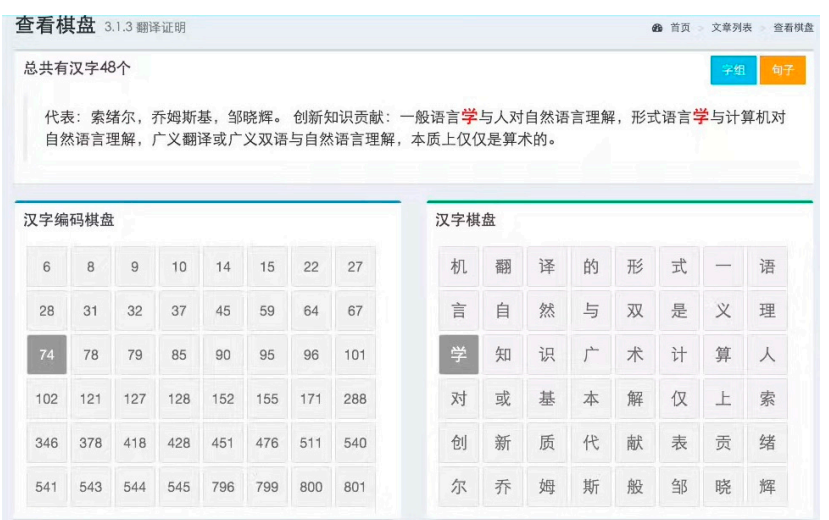

(a)

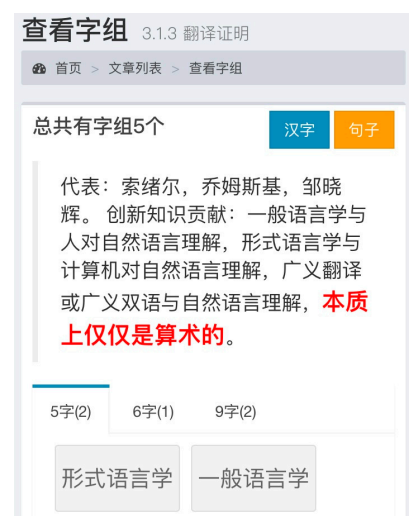

(b)

Figure 3. This is a formal twin chessboard and its application listed as: (a) Description of what is language chessboard; (b) Description of what is knowledge chess-menu and original chess-soul [11].

Table 3. This is a table with chess-menu placed in three rows and columns.

\begin{tabular}{ccc}
\hline Attributes & Concept & Types \\
\hline General & Linguistics & \\
Formal & & Arithmetic \\
Bilingual & Linguistics & Arithmetic \\
\hline
\end{tabular}

\subsection{Three Kinds of Identity: Mathematical Information Law}

The truth is clear. With the aid of the corresponding analytic geometric model and the linkage function, the equivalence relation between the three types of polynomials is given by means of the 
three types of equations of the number, the twins, the text and the solution of the three kinds of identities. In the specific series of the man-machine collaboration process, their constraints were also found.

All numbers and characters should be putted in one of the main twin boards as Figure $4 a, b$.

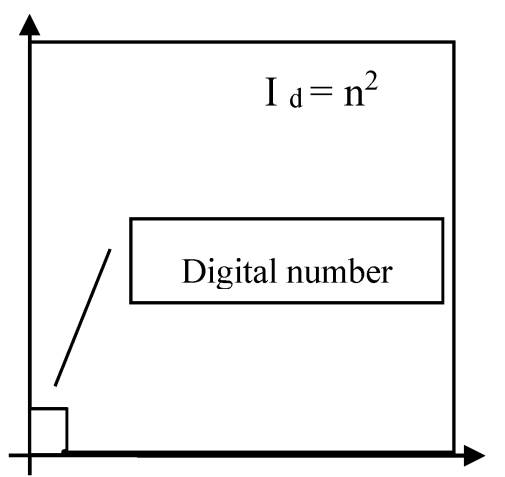

(a)

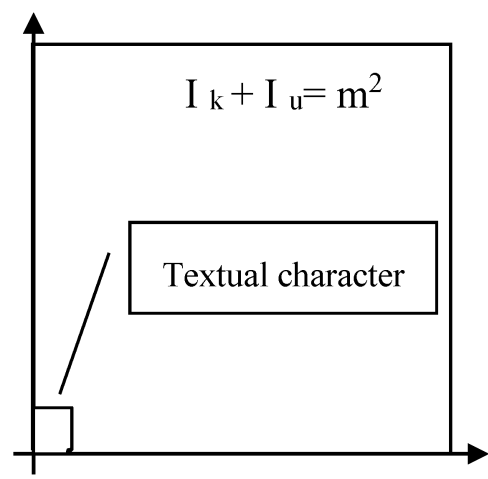

(b)

Figure 4. The same formal matrix both in (a) and (b) listed as: (a) Description of what is contained in the equation $I_{d}=n^{2}$; (b) Description of what is contained in the equation $I_{k}+I_{u}=m^{2}$.

The digital, twins, textual equations:

$$
\begin{gathered}
I_{d}=n^{2} \\
I_{d}=I_{k}+I_{u} \\
I_{k}+I_{u}=m^{2}
\end{gathered}
$$

The above three types of identities, combined with their analytical geometric form, respectively, from the digital, twins, and textual of the three types of character system perspective, from the matrix within the sequence and position of the entire pattern (which, each lattice, have a specific order and position, both mathematically and logically, they are constant, invariant) - thus forming a global reference system that proves the existence of the same mathematical law of information.

(1) The identity of the algebra is not only the solution of the algebraic equation, but also the lattice of the matrix - can be filled in numbers to facilitate the calculation or statistics of the numerator, and the algebraic equation is associated with the geometric pattern, accurately record and express a specific grid in which the specific order and place. (1) This identity records the solution of a particular equation, either a periodic function, a distribution function, or a similar function, whose polynomials represent pure digital relationships. The constraint condition is determined by (2) is ultimately determined by (3).

(2) The identity of the twin, on the one hand, represents the functional relationship between the digital systems, and on the other hand, representing the functional relationship between the text system or the character system, and the performance of the two Linkage function (with twin relationship). (2) is the linkage of the linkage equation, from the linkage function - links (1) and (3) linkage function.

(3) The identity of the text, not only Chinese characters, but also can be the form of English words, or other language system. The relationship between the various character systems, there are strict constraints, usually with the convention of the corresponding relationship or referral as a basis. Thus, (3) the identity, the actual record of the bilingual relationship between the contract is a mixed function - the need for man-machine collaboration to decompose its polynomial (because it is not formal, the need to help indirectly through the linkage function to achieve its formalization).

In this study, the solutions of a series of equations of the three identities can be traced back to their specific equations, functional formulas and polynomials (whether they are numerically or literal, even in the form of twin expressions). 


\section{Conclusions}

The sequence and position of the digital function record, which itself is the only constant on the grid of the matrix. The linkage function is based on the logical and mathematical synonyms (parallel, correspondence, conversion).

Text functions are recorded in the form of Chinese or English words, and the order and position of the lattice in a particular board or matrix are also relatively constant. Although their combination can be ever-changing, but in the linkage function of the constraints, there are still law, follow the rules. This is the role, value and significance of the three types of identities and their corresponding analytic geometric representations. Among them, the three types of identities embody the basic laws of three kinds of information, and the corresponding analytic geometric expression can be presented through the twin chessboard and play a role in the process of man-machine collaboration - which is expressed as expert knowledge acquisition.

Its significance lies in that the Global Positioning System (GPS) should be regarded as a special case of the generalized bilingual system that should be looked like the Global Hardware Positioning System (GHPS) with its Global Software Positioning System (GSPS), Global Language Positioning System (GLPS) and Global Knowledge Positioning System (GKPS).

According to our study and research, we found that: if the twin Turing machine as the twin chessboard with the effect of GSPS, then the man-machine combination reusing as the knowledge chess menu with the effect of GLPS, and further, repeated confirmation, the original chess soul could been created by using GKPS and from its effect.

In other words, for us expert knowledge acquisition, natural language understanding, knowledge expression formalized, and its targeted re-use, can be based on the three basic principles of information by using sequence positioning logic, generalized bilingual mathematics and generalized language translation, and in the corresponding chess board, chess menu and chess soul as the super twin brain development environment -large platform of knowledge production, to a package to resolve.

Acknowledgments: All sources of funding of the study have been disclosed.

Author Contributions: Xiaohui Zou conceived and designed the experiments; Xiaohui Zou performed the experiments and analyzed the data; Xiaohui Zou, Shunpeng Zou, Lijun Ke contributed reagents/materials/analysis tools; Xiaohui Zou wrote the paper.

Conflicts of Interest: The authors declare no conflict of interest.

\section{Appendix A}

Software System: Language Chess Board, Knowledge Chess Menu and Original Chess Soul, the Following are Available Online at:

http://kben.koderx.com/article/63/board and http://kben.koderx.com/article/63/group http://kben.koderx.com/article/64/board and http://kben.koderx.com/article/64/group http://kben.koderx.com/article/65/board and http://kben.koderx.com/article/65/group

\section{References}

1. Beziau, J.-Y. What is "Formal Logic"? In Proceedings of the XXII World Congress of Philosophy, Seoul, Korea, 30 July-5 August 2008; Volume 13, pp. 9-22.

2. Chiswell, I. Mathematical Logic; Oxford University Press: Northamptonshire, UK, 2007.

3. Turing, A.M. Computing Machinery and Intelligence. Mind 1950, 49, 433-460.

4. Searle, J.R. Minds, Brains and Programs. Behav. Brain Sci. 1980, 3, 417-457.

5. Taylor, J.M.; Raskin, V.; Hempelmann, C.F. From Disambiguation Failures to Common-Sense Knowledge Acquisition: A Day in the Life of an Ontological Semantic System. In Proceedings of the 2011 IEEE/WIC/ACM International Conferences on Web Intelligence and Intelligent Agent Technology (WI-IAT 2011), Lyon, France, 22-27 August 2011. 
6. Ludwig, D. Overlapping Ontologies and Indigenous Knowledge. From Integration to Ontological SelfDetermination. Stud. Hist. Philos. Sci. 2016, 59, 36-45.

7. De Saussure, F. Selections from the Course in General Linguistics. In The Continental Philosophy Reader; Kearney, R., Rainwater, M., Eds.; Routledge: London, UK, 1996.

8. Chomsky, N. Syntactic Structures, 2nd ed.; De Gruyter Mouton: Hague, The Netherlands, 1957.

9. Zou, X. Collaborative Intelligent Computing System: Theoretical Model with Its Application. In Proceedings of the 2012 AAAS Annual Meetings, Vancouver, BC, Canada, 16-20 February 2012

10. Zou, X. Basic Law of Information: The Fundamental Theory of Generalized Bilingual Processing. In Proceedings of the SIS Summit Vienna 2015-The Information Society at the Crossroads, Vienna, Austria, 3-7 June 2015.

11. Zou, X. Fundamental Law of Information Proved by Brain and Computer. Online resource: http://blog.sciencenet.cn/home.php?mod=space\&uid=94143\&do=blog\&id=1056171 (accessed on 21 May 2017).

C 2017 by the authors. Licensee MDPI, Basel, Switzerland. This article is an open access article distributed under the terms and conditions of the Creative Commons Attribution (CC BY) license (http://creativecommons.org/licenses/by/4.0/). 